\title{
Magnetism and magnetic asphericity in NiFe alloys
}

\author{
Biplab Sanyal囲 and Abhijit Mookerjee \\ S.N.Bose National Centre for Basic Sciences, JD Block, Sector 3, Salt Lake City, \\ Calcutta 700091, India
}

\begin{abstract}
We here study magnetic properties of $\mathrm{Ni}_{x} \mathrm{Fe}_{1-x}$ using Augmented space recursion technique coupled with tight-binding linearized muffin tin orbital method. Also the spectral properties of this alloy has been studied here.
\end{abstract}

PACS numbers: 71.20.-b,71.23.-k,71.20.Be,71.15.-m

Keywords : Magnetism, alloys, augmented space recursion

\section{Introduction}

$\mathrm{NiFe}$ alloys have been extensively studied earlier. Experimental data have been available for decades. Neutron scattering has probed the local magnetic moments [1, 2, 3, 田, 鴫. Theoretical approaches include the Hartree-Fock approaches of Hasegawa and Kanamori [6] and Kanamori [7]. These authors assumed the density of states to be steeple models, and were therefore only qualitative. They used the coherent potential approach (CPA) of Velický et al [8] in order to describe disorder and configuration averaging. Mishra and Mookerjee [9] have used the Hartree-Fock model of Hasegawa and Kanmori but the averaged density of states was obtained from the augmented space technique based cluster CPA. Podgórny [10] studied NiFe using the supercell linearized muffin-tin orbitals method (LMTO) while Lipiński [1] applied the tight-binding LMTO-CPA.

$\ddagger$ Corresponding author. Fax : 0091 333343477, Phone : 0091 333583061, E-mail : biplab@boson.bose.res.in 
NiFe alloys have interesting behaviour. The magnetic behaviour of $\mathrm{Ni}$ is anomalous in the sense that the local magnetic moment of $\mathrm{Ni}$ depends sensitively on its immediate surrounding. The local magnetic moment on a Ni atom depends upon the number of $\mathrm{Ni}$ neighbours it has. The CPA which replaces the random neighbourhood of an atom in a solid solution by an effective, averaged medium cannot reflect this behaviour. The aim of this communication is to study NiFe by the augmented space recursion (ASR) technique [12]. Recently we have shown that the ASR allows us to go beyond the CPA without violating the essential herglotz analytic properties and take into account the effect of local neighbourhood [13]. The ASR will be based on the TB-LMTO hamiltonian

$$
\begin{aligned}
& H=\sum_{R L} \hat{C}_{R L} \mathcal{P}_{R L}+\sum_{R L} \sum_{R^{\prime} L^{\prime}} \hat{\Delta}_{R L}^{1 / 2} S_{R L, R^{\prime} L^{\prime}} \hat{\Delta}_{R^{\prime} L^{\prime}}^{1 / 2} \mathcal{T}_{R L, R^{\prime} L^{\prime}} \\
& \hat{C}_{R L}=C_{R L}^{B}+\left(C_{R L}^{A}-C_{R L}^{B}\right) n_{R} \\
& \hat{\Delta}_{R L}^{1 / 2}=\Delta_{R L}^{B 1 / 2}+\left(\Delta_{R L}^{A 1 / 2}-\Delta_{R L}^{B 1 / 2}\right) n_{R}
\end{aligned}
$$

Here $\mathcal{P}_{R L}$ and $\mathcal{T}_{R L, R^{\prime} L^{\prime}}$ are projection and transfer operators in the hilbert space spanned by the tight binding basis $|R L\rangle$ and $n_{R}$ is a random occupation variable which is 1 if the site $R$ is occupied by an atom of the A type and 0 if not. The augmented space hamiltonian replaces the random occupation variable by operators $M_{R}$ of rank 2 . For models without any short-range order

$$
\begin{aligned}
M_{R} & =x \mathcal{P}_{\uparrow}^{R}+(1-x) \mathcal{P}_{\downarrow}^{R}+\sqrt{x(1-x)}\left(\mathcal{T}_{\uparrow \downarrow}^{R}+\mathcal{T}_{\downarrow \uparrow}^{R}\right) \\
|\uparrow\rangle & =(\sqrt{x}|0\rangle+\sqrt{1-x}|1\rangle) \\
|\downarrow\rangle & =(\sqrt{1-x}|0\rangle-\sqrt{x}|1\rangle)
\end{aligned}
$$

The ASR was carried out with LDA self-consistency. The computations included scalar relatavistic corrections. The exchange potential of von Barth-Hedin was used. We 
used the choice of flexible Wigner Seitz radii for Ni and Fe as suggested by Kudrnovský and Drchal [14] in order to ensure the atomic spheres to be neutral and avoid the calculation of the Madelung energy.

The recursion method then expresses the Green functions as continued fraction expansions. The continued fraction coefficients are exactly obtained upto eight levels and the terminator suggested by Luchini and Nex 15] is used to approximate the asymptotic part. The convergence of this procedure has been discussed by Ghosh et al [16]. The local charge densities are given by :

$$
\rho_{\sigma}^{\lambda}(r)=(-1 / \pi) \Im m \sum_{L} \int_{-\infty}^{E_{F}} d E \ll G_{L L}^{\lambda, \sigma}(r, r, E) \gg
$$

Here $\lambda$ is either $A$ or $B$. The local magnetic moment is

$$
m^{\lambda}=\int_{r<R_{W S}} d^{3} r\left[\rho_{\uparrow}(r)-\rho_{\downarrow}(r)\right]
$$

We have also obtained the spectral densities and complex band structures for 50-50 Ni-Fe using the ASR in k-space as suggested by Biswas et al [17].

For high concentrations of $\mathrm{Ni}$ the alloy forms a face centred cubic solid solution. With decreasing Ni concentration, at the invar concentration of about $30 \%$, the system undergoes a structural phase transition to a body centred cubic solid solution. At this transition there is a sharp decrease of Wigner-Seitz radius. This leads to larger overlap of the $d$-bands, and hence, using the Stoner criterion, a decrease of magnetization. We shall limit ourselves to the face centred cubic region beyond the invar concentration.

\section{Results and Discussion}

Figures 1(a) and (b) show the Ni and Fe partial density of states for Ni concentrations varying between 90 and $40 \%$. We note that the Ni densities hardly change with concentration, while the Fe densities change considerably. For the minority spin partial 
densities on Fe, the structures below the Fermi energy do not change with concentration. The peak at around 0.0 Ryd grows with increasing Ni concentration. However, this structure is above the Fermi level and does not contribute to the magnetic moment. For the majority spin partial densities on Fe, the peak around -0.4 Ryd grows with increasing $\mathrm{Ni}$ concentration . This ensures that the Ni local magnetic moment remains almost concentration independent, while the Fe local moment increases with Ni concentration. Figure 2 shows the $\mathrm{Ni}$ and Fe moments as well as the average moment as functions of Ni concentration. The results agree rather well with earlier CPA results. In addition we have shown the experimental results on the average magnetic moment by Crangle and Halam [18 as well as the experimental data on local magnetic moments on $\mathrm{Fe}$ and $\mathrm{Ni}$ by Shull and Wilkinson [1], Collins et al [2] and Nishi et al [3]. The experimental data are reasonably reproduced, as well as CPA did earlier.

Both the CPA and our results indicate a slight increase of the Fe magnetic moment as the Ni concentration increases. The experimental data with its large error bars tell us little about this trend with certainty. The asphericity, which is measured by the ratio of the $t_{2 g}$ and $e_{g}$ contributions to the magnetic moment, is shown in figure 3 . The results indicate that the Ni moment distribution is highly anisotropic. The $t_{2 g}$ dominates the magnetization as well as the density of states peaks near the Fermi level. We also compare our results with neutron scattering experimental data of Brown et al [4] and Ito et al [5]. The asphericity trend with concentration is reproduced, but our average asphericities are slightly larger. The asphericity decreases with decreasing Ni concentration. The Fe moment distribution is almost spherical throughout the concentration range, with a slight decrease with decreasing $\mathrm{Ni}$ concentration. The average asphericity agrees reasonably well with experiment [4, 5].

Figures 4 (a) and (b) gives the spectral densities for k-vectors going from the $\Gamma$ to 
the $X$ point for the $e_{g}$ and $t_{2 g}$ bands. The results clearly show the splittng between the majority and minority spin bands. The imaginary part of the self-energy which measures the disorder scattering life-time, is clearly k-dependent and is more prominent at the $X$ point and least at the $\Gamma$ point. This is in contrast to the CPA calculations where the life-times are almost k-independent. Angle-resolved photoemmision experiments show the spectral behaviour of alloys and the nature of fuzzy fermi surfaces can be obtained from Compton scattering and positron annihilation experiments. The complex bands are obtained from the peaks and widths in the spectral densities. These are shown in figure 5 (a) and (b). We note that the d-bands of Fe and Ni overlap considerably, and although the $e_{g}$ and $t_{2 g}$ type bands are evident, they are broadened by disorder. The lines shown in figure 5 are to guide the eye. The statements made above based on the spectral functions are clearly seen in this figure. The Fermi level crosses the minority spin bands. These bands have considerable life-times and the Fermi surface should have this width associated with it. It should be interesting to look at the fuzzy Fermi surface experimentally.

NiFe alloys tend to exhibit short-ranged order. The augmented space recursion is ideally suited to take into account effects of short-ranged order. The formalism to include this has been introduced earlier by Mookerjee and Prasad [19] and applied to alloy systems by Saha et al [13]. We have carried out the calculation of the magnetic moment of the 50-50 NiFe alloy as a function of the Warren-Cowley short-ranged order parameter $\alpha$. The magnetic moment of Fe is hardly affected by short-ranged order. However, in the region where $\alpha<0$ indicating ordering, $\mathrm{Ni}$ is more likely to have $\mathrm{Fe}$ neighbours as compared to the case without short-ranged order. Here the magnetic moment on $\mathrm{Ni}$ increases. Similarly, when $\alpha>0$, Ni atoms segregate and are less likely to have $\mathrm{Fe}$ as their neighbours and the Ni moment decreases. This is shown in figure 
6. We conclude that extra moment is induced on $\mathrm{Ni}$ by Fe atoms in its vicinity. This induced moment on $\mathrm{Ni}$ is most sensitive to short-ranged order in the alloy.

\section{References}

[1] Shull C G and Wilkinson M K 1955 Phys. Rev. 97304

[2] Collins M F, Jones R V and Lowde R D 1962 J. Phys. Soc. Japan 17 B-III 19

[3] Nishi M, Nakai Y and Kunitomi N 1974 J. Phys. Soc. Japan 37570

[4] Brown P J, Deportes J and Ziebeck K R A 1991 J. Physique 11529 ; Brown P J, Jassim I K, Neumann K U and Ziebeck K R A 1989 Physica B161 9

[5] Ito Y, Akimitsu J, Matsui M and Chikazami S 1979 J. Magn. Magn. Mat. 10194.

[6] Hasegawa H and Kanamori J 1971 J. Phys. Soc. Japan 31382 ; 1972 J. Phys. Soc. Japan 331599

[7] Kanamori J 1963 Prog. Theor. Phys. 30275

[8] Velický B, Kirkpatrick S and Ehrenreich H 1968 Phys. Rev. 175747

[9] Mishra A K and Mookerjee A 1990 Int. J. Mod. Phys. B4 605

[10] Podgorný P 1990 Acta Phys. Polon. A78 941

[11] Lipiński S 1993 Int. J. Mod. Phys. B7 965

[12] Mookerjee A 1973 J. Phys. C: Solid State Phys. 6 L205; 6 1340; Haydock R, Heine V and Kelly M J 1972 J. Phys. C: Solid State Phys. 52845

[13] Saha T, Dasgupta I and Mookerjee A 1996 J. Phys.: Condens. Matter 81979

[14] Kudrnovský J and Drchal V 1990 Phys. Rev. B41 7515

[15] Luchini M U and Nex C M M 1987 J. Phys. C: Solid State Phys. 203125

[16] Ghosh S, Das N and Mookerjee A 1997 J. Phys.: Condens. Matter 91701

[17] Biswas P , Sanyal B, Fakhruddin M, Halder A, Mookerjee A and Ahmed M 1995 J. Phys.: Condens. Matter 78569

[18] Crangle J and Halam G C 1963 Proc. Roy. Soc. A272 119

[19] Mookerjee A and Prasad R 1993 Phys. Rev. B48 17724

\section{Figure Captions}

Figure 1 (a) Partial densities of states at Ni sites. (b) Partial densities of states at Fe sites. The concentrations of $\mathrm{Ni}$ are shown as are the Fermi energies (vertical lines).

Figure 2 Magnetic moments on $\mathrm{Fe}$ and $\mathrm{Ni}$ sites and the averaged magnetic moment as functions of $\mathrm{Ni}$ concentration. The diamond marks indicate the experimental results of Crangle and Halam, the dashed points of Shull and Wilkinson, the square points of Collins and Lowde and the crossed points of Nishi et al .

Figure 3 Asphericity of moment distribution on $\mathrm{Fe}$ and $\mathrm{Ni}$ sites as functions of $\mathrm{Ni}$ 
concentration. The cross marks indicate the neutron scattering results of Brown et $a l$ and Ito et al .

Figure 4 The spectral densities for (a) $e_{g}$ and (b) $t_{2 g}$ states along the $\Gamma$ to $X$ direction for 50-50 FeNi. The bold curves are for the majority spins and the dotted curve for the minority spins. For both (a) and (b), the wave vectors of figures are (from top to bottom) $(1,0,0),(0.75,0,0),(0.5,0,0),(0.25,0,0)$ and $(0,0,0)$ in units of $2 \pi / a$, where $\mathrm{a}$ is the lattice constant.

Figure 5 Complex band structure for $d$-bands of 50-50 FeNi (a) For the majority spin bands (b) for the minority spin bands. The widths of the bands are marked and the lines are only to guide the eye.

Figure 6 The averaged magnetic moment and the magnetic moment of $\mathrm{Ni}$ as functions of the Warren-Cowley short-ranged order parameter for 50-50 NiFe. 
Figure 1(a)

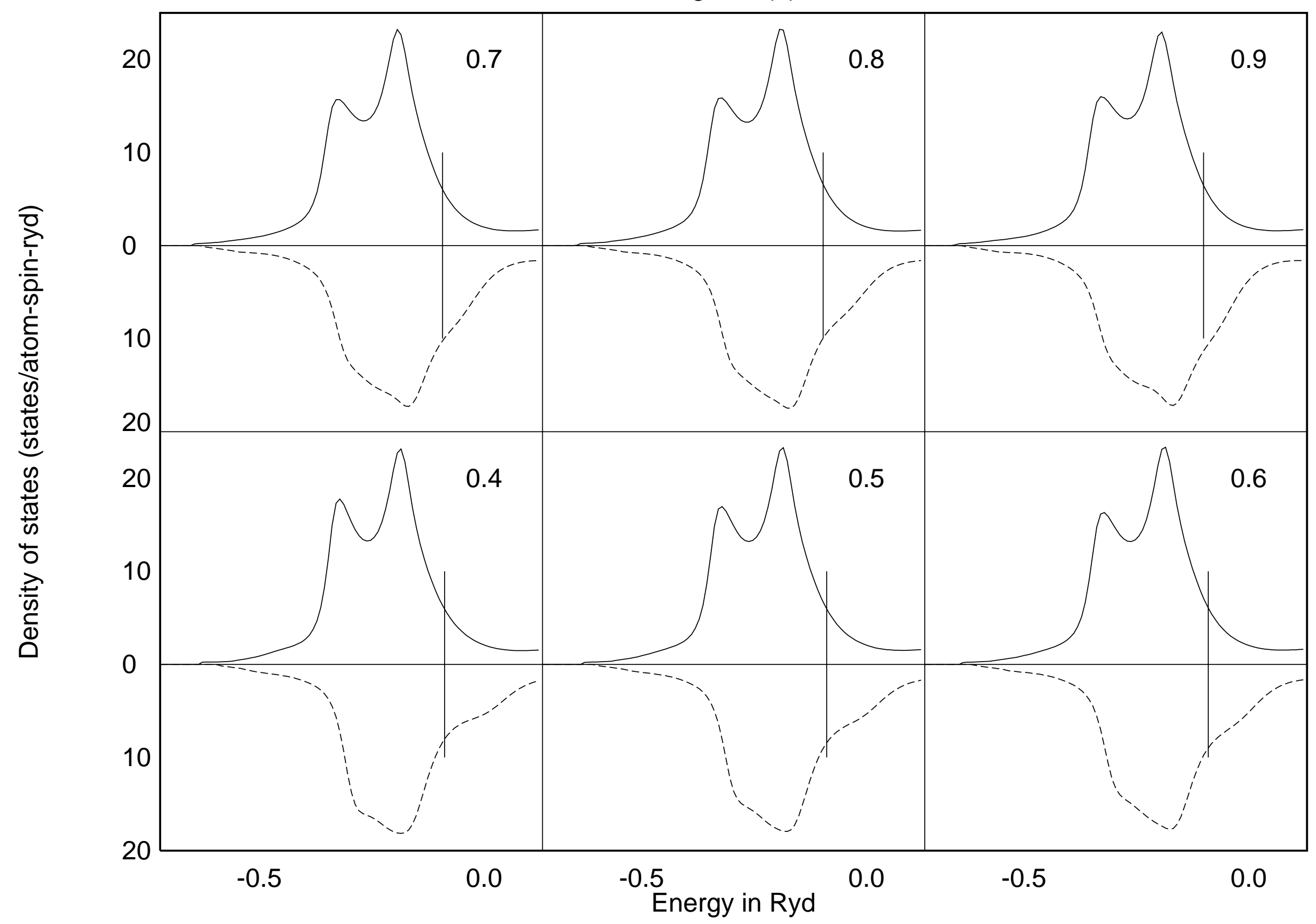


Figure 1(b)

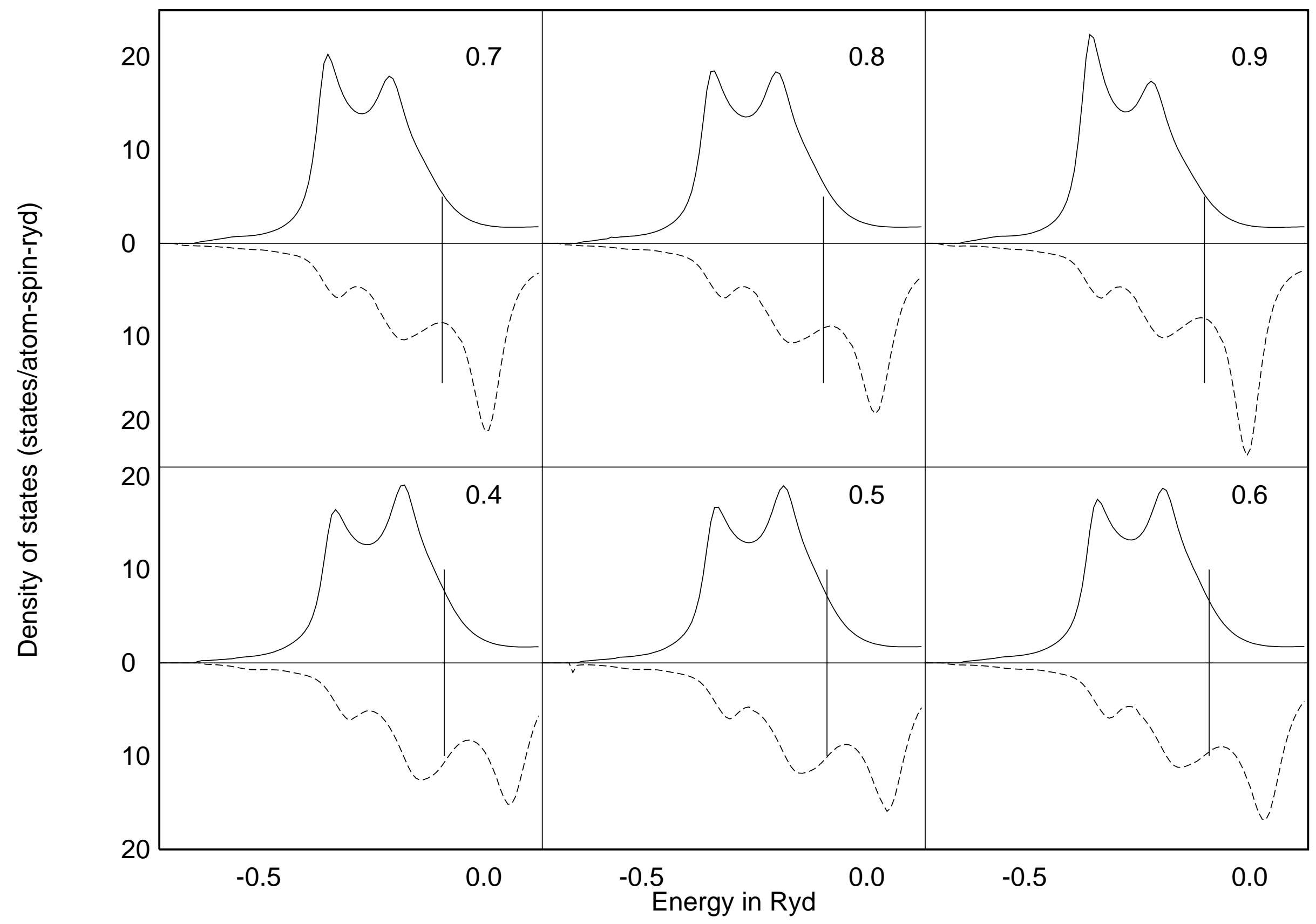


Figure 1(b)

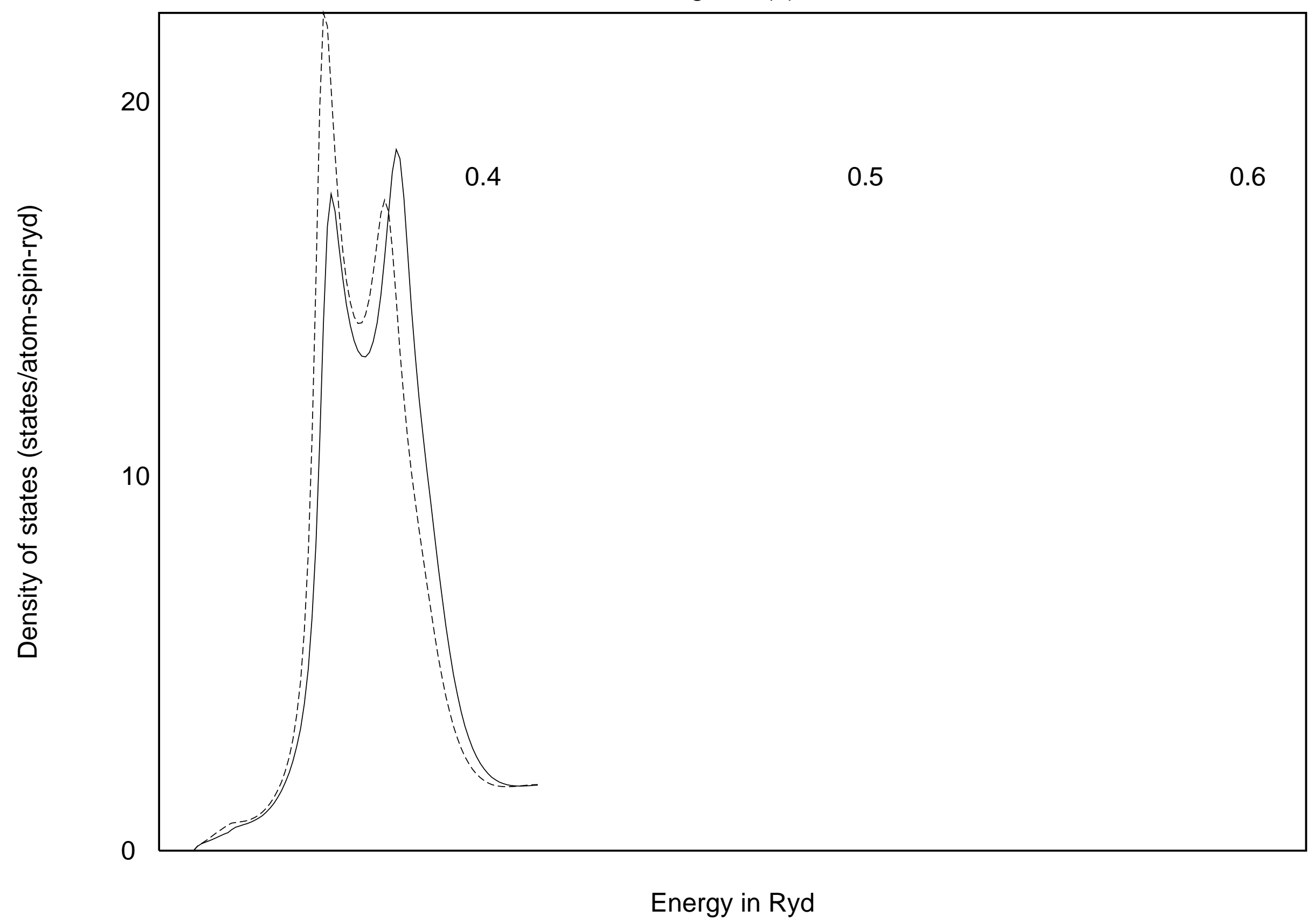


Figure 2

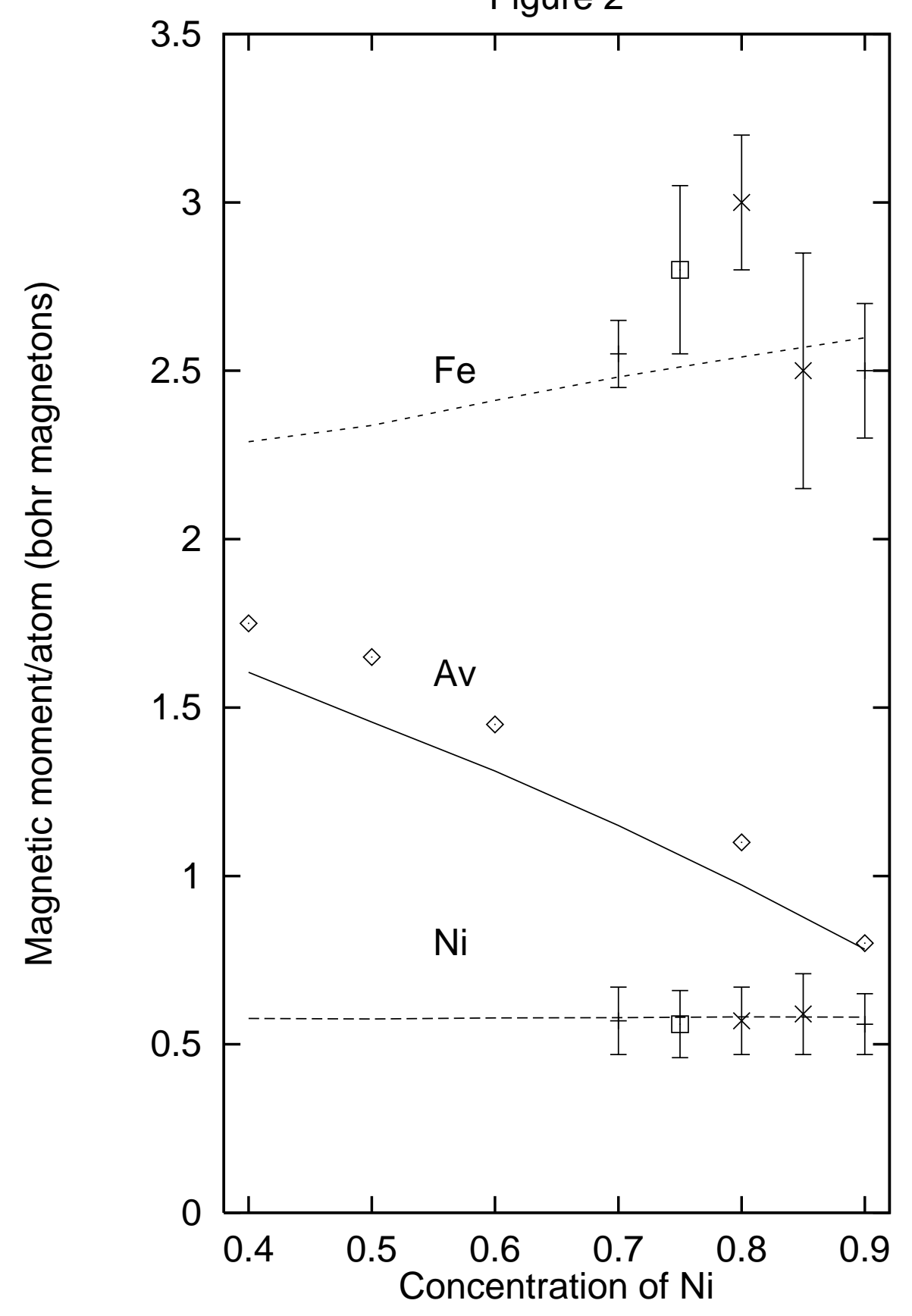


Figure 3

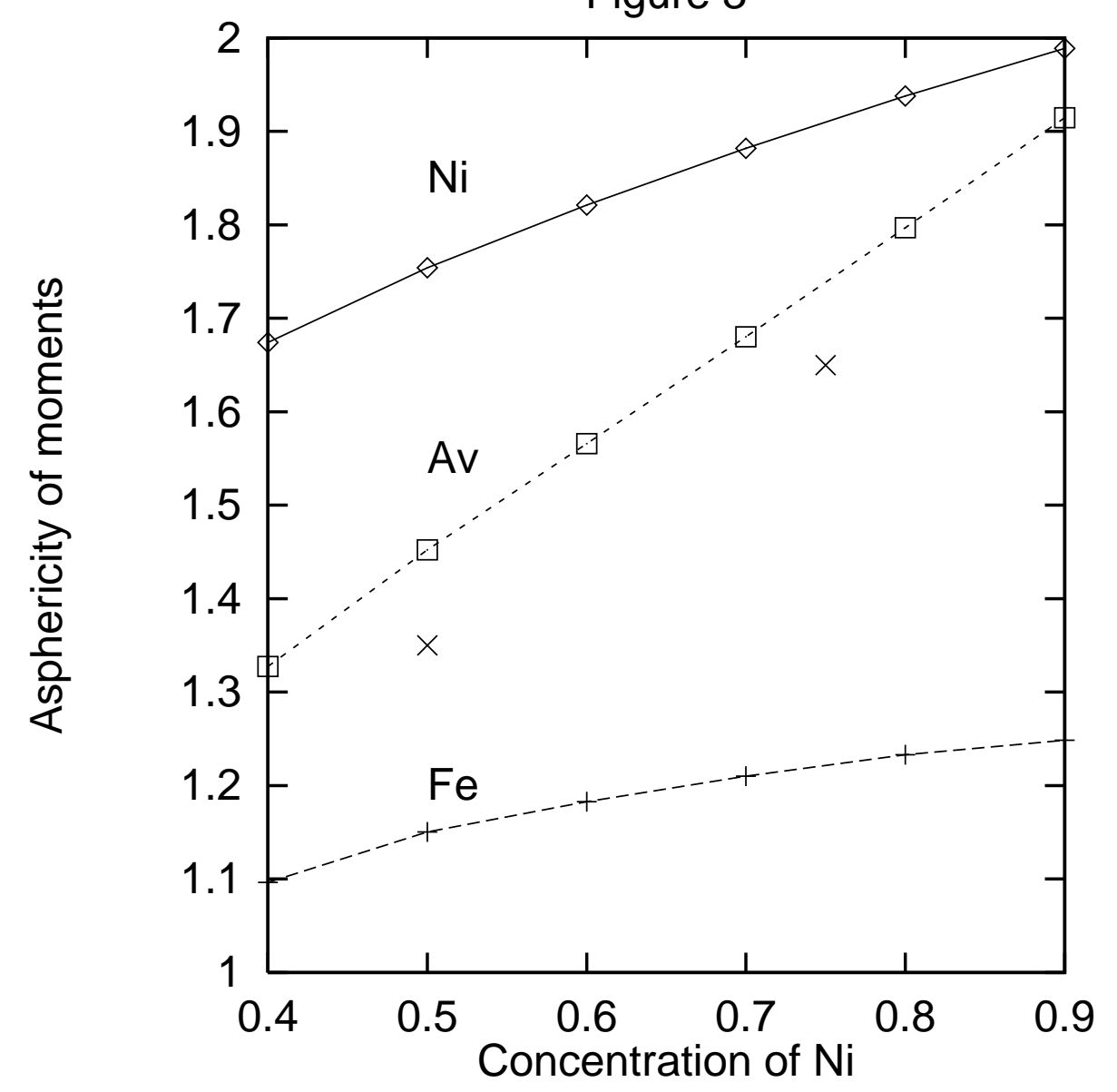




\section{Spectral density (arbitrary units)}

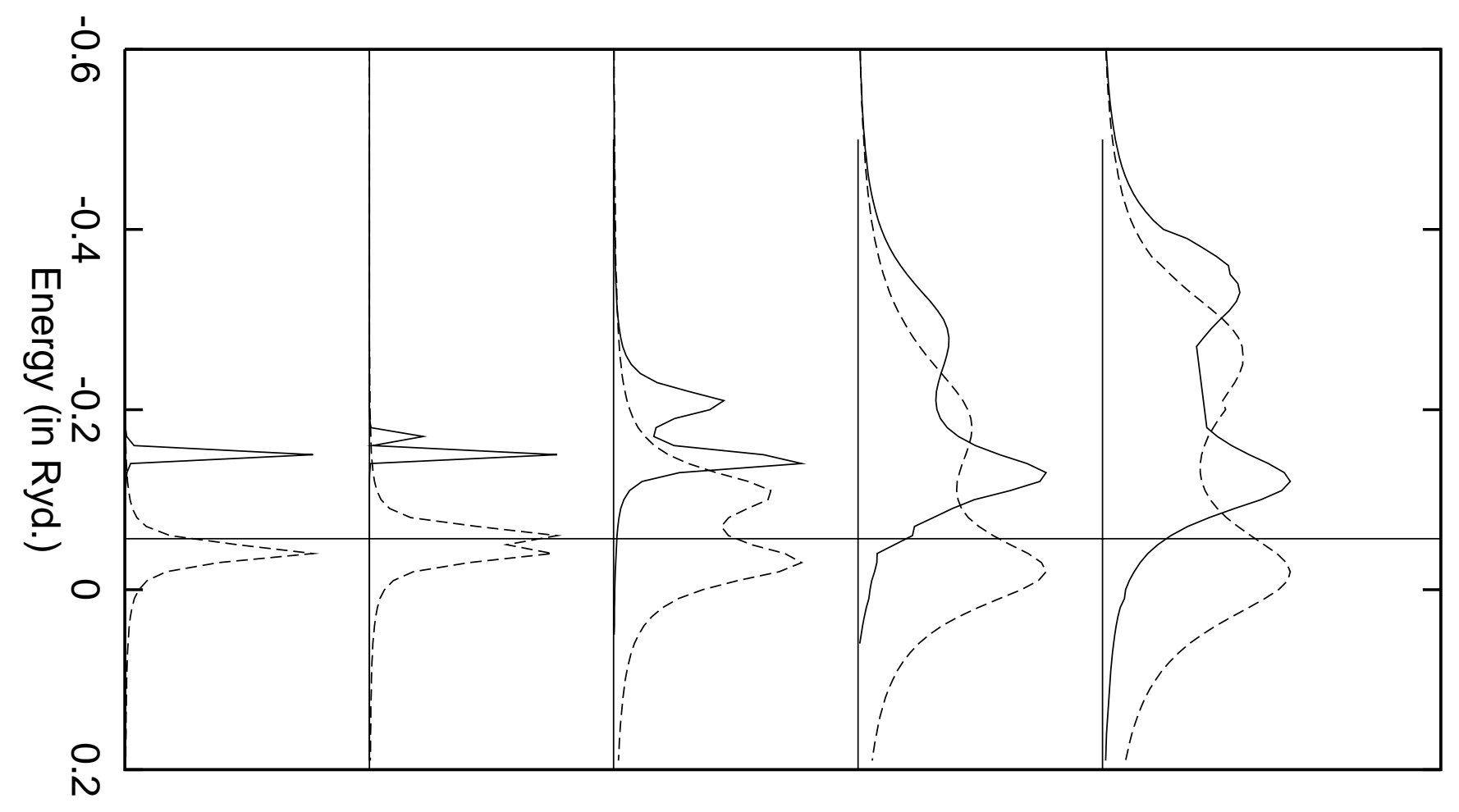


Spectral density (arbitrary units)

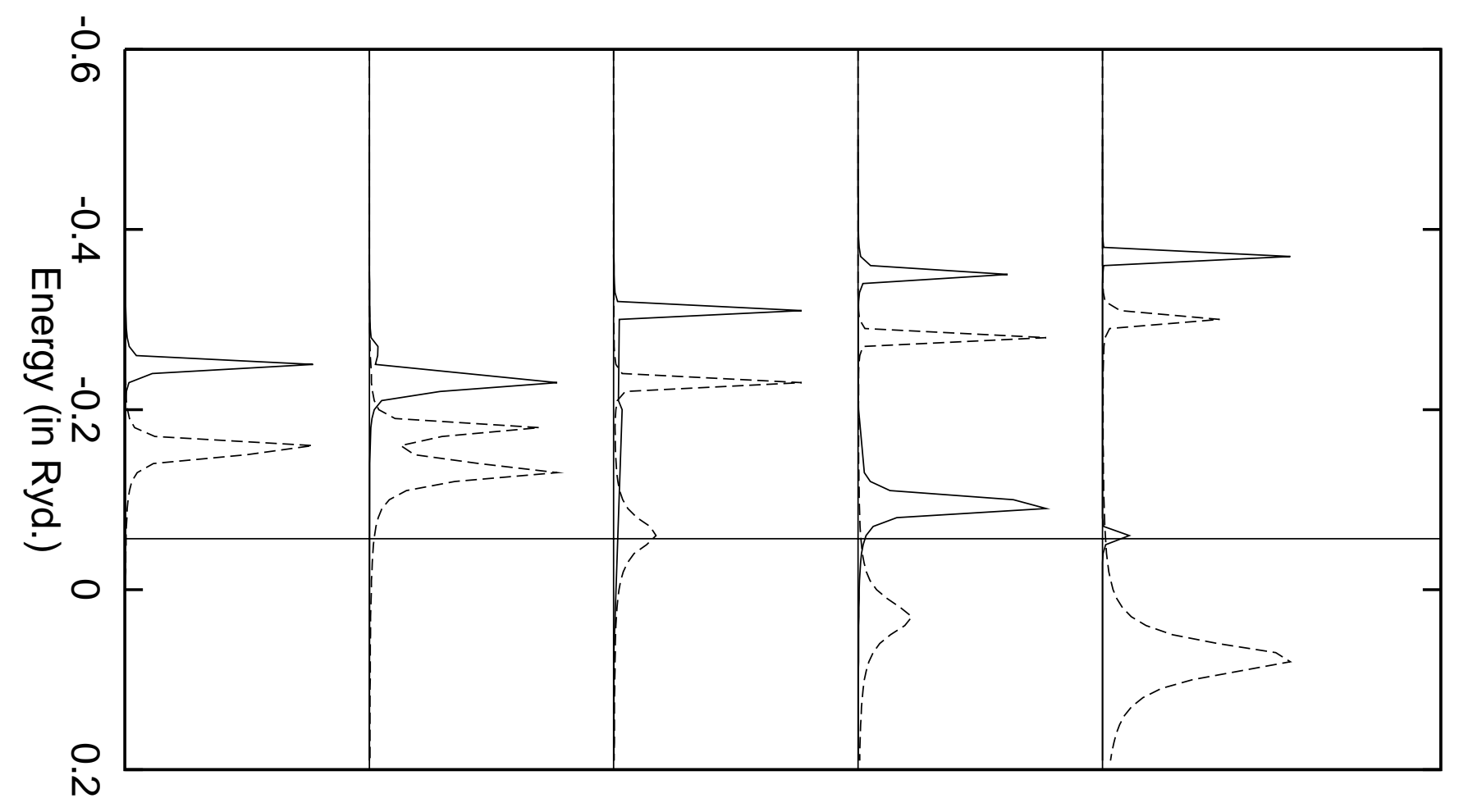


Figure 5(a)

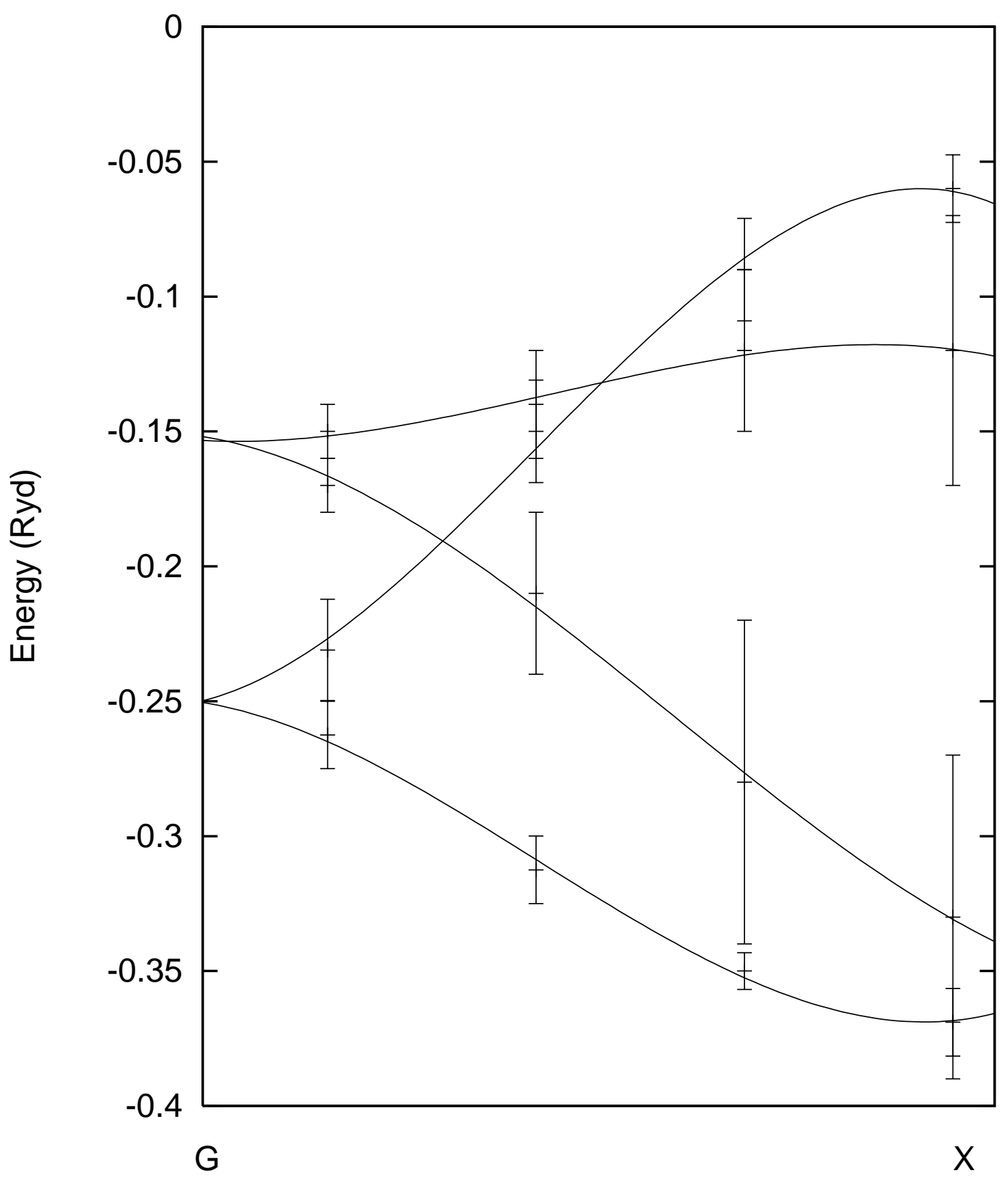


Figure 5(b)

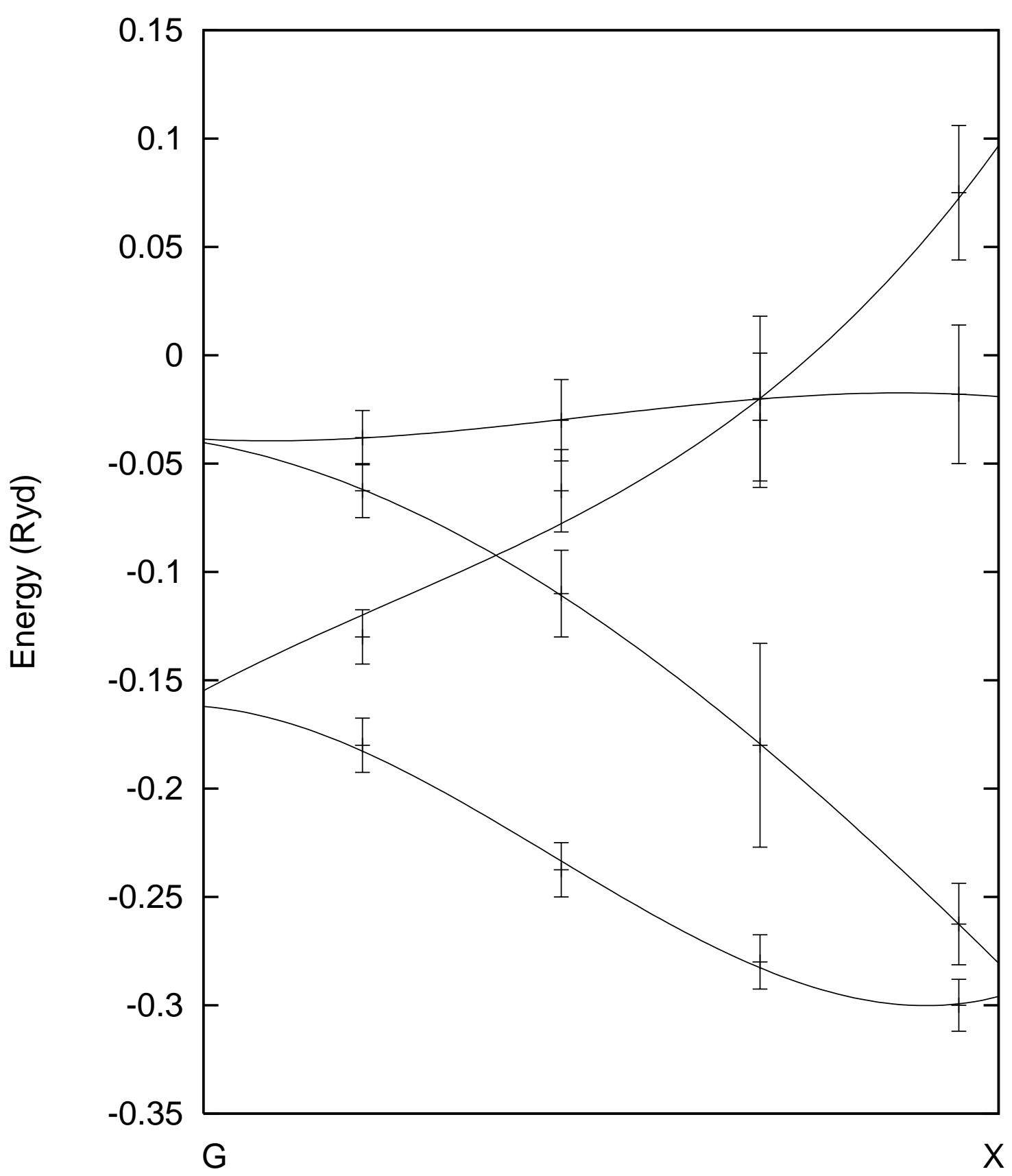


Figure 6

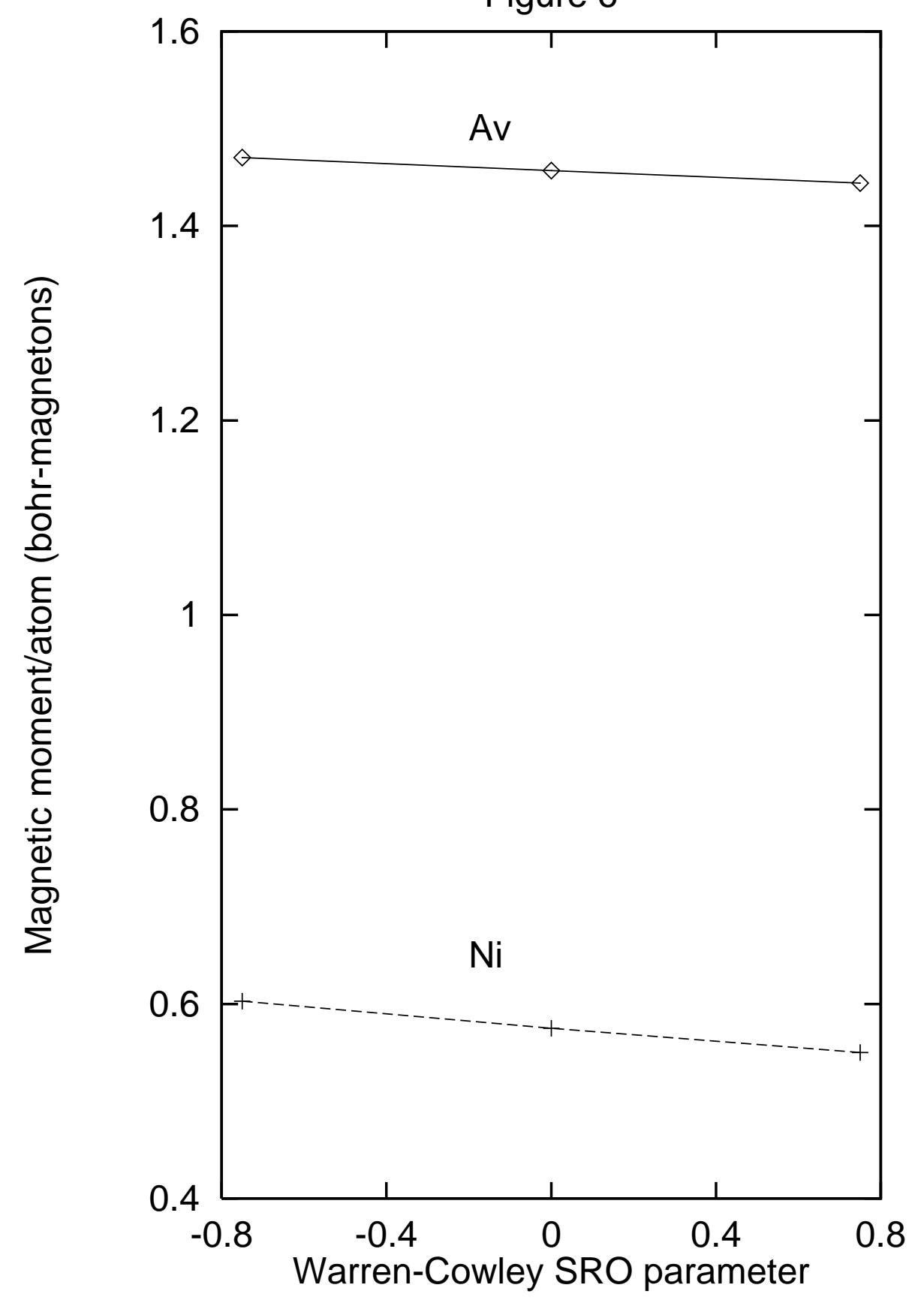

